\title{
PRICING OF CATASTROPHE INSURANCE OPTIONS UNDER IMMEDIATE LOSS REESTIMATION
}

\author{
FRANCESCA BIAGINI*** AND \\ YULIYA BREGMAN, ${ }^{* * * *}$ Ludwig-Maximilians Universität München \\ THILO MEYER-BRANDIS, ${ }^{* * * *}$ University of Oslo
}

\begin{abstract}
We specify a model for a catastrophe loss index, where the initial estimate of each catastrophe loss is reestimated immediately by a positive martingale starting from the random time of loss occurrence. We consider the pricing of catastrophe insurance options written on the loss index and obtain option pricing formulae by applying Fourier transform techniques. An important advantage is that our methodology works for loss distributions with heavy tails, which is the appropriate tail behavior for catastrophe modeling. We also discuss the case when the reestimation factors are given by positive affine martingales and provide a characterization of positive affine local martingales.
\end{abstract}

Keywords: Catastrophe insurance option; loss index; Fourier transform; option pricing formula; heavy tail; positive affine martingale

2000 Mathematics Subject Classification: Primary 46N30; 60G07; 91B30

\section{Introduction}

Over the past decades the rise in insured losses has exploded from 2.5 billion dollars per year to an average value of the aggregated insurance losses of 30.4 billion dollars per year (2006 prices); see [20] for more details. Table 1 gives a summary of the 10 most expensive natural catastrophes for the last 30 years.

In order to securitize the catastrophe risk, insurance companies have tried to take advantage of the vast potential of capital markets by introducing exchange-traded catastrophe insurance options. Exchange-traded insurance instruments present several advantages with respect to reinsurance, for example, they offer low transaction costs, because they are standardized, and include minimal credit risk, because the obligations are guaranteed by the exchange. See [18] and [19] for the comparison of insurance securities. In particular, catastrophe options are standardized contracts based on an index of catastrophe losses, for example, compiled by the Property Claim Service (PCS), an internationally recognized market authority on property losses from catastrophes in the US.

The first index-based catastrophe derivatives were introduced at the Chicago Board of Trade in 1992, but there was only little activity in the market. A second version of the index, compiled by the PCS, was introduced in 1995 . At the peak, the total capacity created by this version

Received 28 February 2008; revision received 18 July 2008.

* Postal address: Department of Mathematics, Ludwig-Maximilians Universität München, Theresienstrasse 39, D-80333 Munich, Germany.

** Email address: biagini@mathematik.uni-muenchen.de

*** Email address: bregman@mathematik.uni-muenchen.de

**** Postal address: CMA, University of Oslo, Postbox 1035, Blindern, Norway. Email address: meyerbr@math.uio.no 
TABLE 1: Top 10 insured catastrophe losses (source: Swiss Re, sigma Nr. 2/2007).

\begin{tabular}{|c|c|c|c|}
\hline $\begin{array}{l}\text { Insured loss } \\
\left(\times 10^{9} \text { dollars }\right)\end{array}$ & Year & Event & Country \\
\hline 66.3 & 2005 & $\begin{array}{l}\text { Hurricane Katrina: } \\
\text { floods } \\
\text { dams burst } \\
\text { damage to oil rigs }\end{array}$ & $\begin{array}{l}\text { US } \\
\text { Gulf of Mexico } \\
\text { Bahamas } \\
\text { North Atlantic }\end{array}$ \\
\hline 23.0 & 1992 & $\begin{array}{l}\text { Hurricane Andrew: } \\
\text { flooding }\end{array}$ & $\begin{array}{l}\text { US } \\
\text { Bahamas }\end{array}$ \\
\hline 21.4 & 2001 & $\begin{array}{l}\text { Terrorist attack on World Trade Center, } \\
\text { Pentagon, and other buildings }\end{array}$ & US \\
\hline 19.0 & 1994 & Northridge earthquake & US \\
\hline 13.7 & 2004 & $\begin{array}{l}\text { Hurricane Ivan: } \\
\text { damage to oil rigs }\end{array}$ & $\begin{array}{l}\text { US } \\
\text { Caribbean }\end{array}$ \\
\hline 13.0 & 2005 & $\begin{array}{l}\text { Hurricane Wilma: } \\
\text { torrential rain } \\
\text { floods }\end{array}$ & $\begin{array}{l}\text { US } \\
\text { Mexico } \\
\text { Jamaica } \\
\text { Haiti }\end{array}$ \\
\hline 10.4 & 2005 & $\begin{array}{l}\text { Hurricane Rita: } \\
\text { floods } \\
\text { damage to oil rigs }\end{array}$ & $\begin{array}{l}\text { US } \\
\text { Gulf of Mexico } \\
\text { Cuba }\end{array}$ \\
\hline 8.6 & 2004 & Hurricane Charley & $\begin{array}{l}\text { US } \\
\text { Caribbean }\end{array}$ \\
\hline 8.4 & 1991 & Typhoon Mireille & Japan \\
\hline 7.4 & 1989 & Hurricane Hugo & $\begin{array}{l}\text { US } \\
\text { Puerto Rico }\end{array}$ \\
\hline
\end{tabular}

of the PCS options amounted to 89 million. Trading in these options slowed down in 1999. In a separate initiative, the Bermuda Commodities Exchange (BCE) was launched in 1997 to trade property catastrophe-linked option contracts. The BCE suspended its operations in 1997. Trading in the PCS options slowed down in 1999, because of the lack of market liquidity and of qualified personnel (see, e.g. [18]).

However, the record losses caused by hurricanes Katrina, Rita, and Wilma in 2005 have been a catalyst in creating new derivative instruments to trade the catastrophe risks in the capital markets. In March 2007, the New York Mercantile Exchange (NYMEX) began trading catastrophe futures and options again. These new contracts were designed to bring the transparency and liquidity of the capital markets to the insurance sector, providing effective ways of protecting against property catastrophe risk and providing the investors with the opportunity to trade a new asset class which has little or no correlation to other exchange-traded asset classes. The NYMEX catastrophe options are settled against the Re-Ex loss index, which is created from the data supplied by the PCS.

Following the descriptions in [12], [17], and [18], the structure of catastrophe insurance options is described as follows. The options are written on a loss index that evolves over two periods: the loss period $\left[0, T_{1}\right]$ and the consecutive development period $\left[T_{1}, T_{2}\right]$. During the contract specific loss period, the index measures catastrophic events that may occur. However, at the time of catastrophe occurrence, the reported losses are only estimates of the true losses, and these estimates are consecutively reestimated until the end, $T_{2}$, of the development period. 
The loss index thus provides, at any $t \in\left[0, T_{2}\right]$, an estimation of the accumulation of the final time $\left(T_{2}\right)$ amounts of catastrophe losses that have occurred during the loss period. Let $N_{t}, t \in$ $\left[0, T_{1}\right]$, denote the number of catastrophes up to time $t$, and let $U_{i}, i=1, \ldots, N_{t}$, denote the corresponding final amounts of losses at time $T_{2}$ (which are unknown at time $0 \leq t<T_{2}$ ). Then the value $L_{t}$ of the loss index can be expressed as

$$
L_{t}=\sum_{i=1}^{N_{t \wedge T_{1}}} \mathrm{E}\left[U_{i} \mid \mathcal{F}_{t}\right], \quad t \in\left[0, T_{2}\right],
$$

where the filtration $\left\{\mathcal{F}_{t}, t \in\left[0, T_{2}\right]\right\}$ represents the information available. If the number $N_{t}$ of catastrophes is assumed to follow a Poisson process, the structure of the index is thus a compound Poisson sum with martingales as summands.

In the literature, a few models have been proposed in order to model the catastrophe loss index and to price catastrophe options written on this index. In [12], [13], and [14] the underlying catastrophe index was represented as a compound Poisson process with nonnegative jumps. In this model, reestimation is not taken into account at all. In [2] and [11] the authors distinguished between a loss and a reestimation period and modeled the index as an exponential Lévy process over each period. However, reestimation is assumed to start exactly at $T_{1}$ by a common reestimation factor. This assumption is not realistic because loss reestimation happens individually for each catastrophe and begins almost immediately after the catastrophic event. In addition, the assumption of an exponential model for accumulated losses during the loss period is rather unrealistic. For example, this implies that later catastrophes are more severe than earlier catastrophes, and that the index starts at a positive value (instead of starting at 0 ). In [1] a more realistic model for the loss index was proposed and analytical catastrophe option pricing formulae were developed, but reestimation was also done by a common factor over the development period only. In [16] a model including immediate reestimation was assumed, where the reestimation was modeled through individual reestimation factors given by geometric Brownian motion. However, no efficient pricing methods were obtained.

In this paper we specify a realistic model for the loss index that is consistent with (1.1). As a particular case, it comprises the model proposed in [16]. We assume that catastrophe occurrence is modeled by a Poisson process, and consider individual reestimation for each catastrophe where the initial estimate of the $i$ th catastrophe loss is reestimated immediately by a positive martingale starting from the random time of loss occurrence. We then consider the pricing of catastrophe options written on the index. The main contribution of this paper is to employ Fourier transform techniques in order to obtain option pricing formulae. To this end, we manage to reduce the calculation of the characteristic function of the index to the computation of an expectation of the characteristic function of the reestimation factor, evaluated in two independent random variables. We mention, in particular, that our methodology also works for loss distributions with heavy tails, which is the appropriate tail behavior for catastrophe modeling. We then proceed to discuss the case when the reestimation factors are given by positive affine martingales. In this situation we provide a characterization of positive affine (local) martingales.

We believe that the use of exchange-traded insurance derivatives will play a crucial role in the securitization of the increasing catastrophe risk in the future. For this purpose, one essential task is to develop quantitative tools that help to establish liquid trading of these instruments. We hope that this paper contributes to this aim in that it sets new insights in the pricing of catastrophe options. 
The remaining parts of the paper are organized as follows. In Section 2 we present the model for the loss index. In Section 3 we consider the pricing of European options in the general model by using Fourier transform techniques, and in Section 4 we discuss the special case of positive affine martingales as reestimation factors. We conclude with Section 5, where we explicitly compute prices for spread options, which are the typical instruments in the market.

\section{Modeling the loss index}

Let $(\Omega, \mathcal{F}, \mathrm{P})$ be a complete probability space. We consider a financial market endowed with a risk-free asset with deterministic interest rate $r_{t}$, and the possibility of trading catastrophe insurance options, written on a loss index. Following the description in the introduction, we distinguish two time periods:

- a loss period $\left[0, T_{1}\right]$;

- a development period $\left[T_{1}, T_{2}\right], T_{1}<T_{2}<\infty$.

During the contract specific loss period $\left[0, T_{1}\right]$, the catastrophic events occur. After the loss period, option users can choose either a six month or a twelve month development period $\left[T_{1}, T_{2}\right]$, where the reestimates of catastrophe losses that occurred during the loss period continue to affect the index. The option contract matures at the end of the chosen development period $T_{2}$.

Motivated by the index structure (1.1) discussed in the introduction, we model the stochastic process $L=\left(L_{t}\right)_{0 \leq t \leq T_{2}}$, representing the loss index as follows:

$$
L_{t}=\sum_{j=1}^{N_{t \wedge T_{1}}} Y_{j} A_{t-\tau_{j}}^{j}, \quad t \in\left[0, T_{2}\right],
$$

where the following notation and hypotheses have been used.

(H1) $N_{s}, s \in\left[0, T_{2}\right]$, is a Poisson process with intensity $\lambda>0$ and jump times $\tau_{j}$ that models the number of catastrophes occurring during the loss period.

(H2) $Y_{j}, j=1,2, \ldots$, are positive independent and identically distributed (i.i.d.) random variables with distribution function $F^{Y}$ that represent the first loss estimation at the time the $j$ th catastrophe occurs.

(H3) $A_{s}^{j}, s \in\left[0, T_{2}\right]$, and $j=1,2, \ldots$, are positive i.i.d. martingales such that

$$
A_{0}^{j}=1 \quad \text { for all } j=1,2, \ldots
$$

(H4) $A^{j}, Y_{j}, j=1,2, \ldots$, and $N$ are independent.

In the sequel we will drop the index $j$ and simply write $Y$ and $A$ in some formulae, when only the probability distribution of the objects matters.

The martingales $A_{t}^{j}$ represent the unbiased reestimation factors. Here we assume that reestimation begins immediately after the occurrence of the $j$ th catastrophe with initial loss estimate $Y_{j}$, individually for each catastrophe.

Here we suppose that market participants observe the evolution of the individual catastrophe losses. Note that observing the market quotes of the catastrophe index $L$ alone is in general not sufficient for the knowledge of the single reestimation factors, $A$. However, it might not be 
unrealistic to assume that market participants are able to obtain additional information about the evolution of individual catastrophes. Therefore, we assume that the market information filtration $\left(\mathcal{F}_{t}\right)_{0 \leq t \leq T_{2}}$ is the right continuous completion of the filtration generated by the catastrophe occurrences $N$, the corresponding initial loss estimates $Y_{j}$, and the corresponding reestimation factors $A^{j}$.

\section{Pricing of insurance derivatives}

We now consider the problem of pricing a European option with payoff depending on the value $L_{T_{2}}$ of the loss index at maturity $T_{2}$. In the catastrophe insurance market, the underlying index $L$ is not traded. Hence, the market is highly incomplete and the choice of the pricing measure is not clear.

Here we suppose the common approach that the risk neutral pricing measure is structure preserving for the model, except for the fact that the pricing measure might introduce a drift into the reestimation martingales $A^{j}, j=1,2, \ldots$ Here we do not discuss further the choice of the pricing measure, and, without loss of generality, perform pricing with the model specification given under $\mathrm{P}$, where we substitute hypothesis (H3) with

$\left(\mathrm{H}^{\prime}\right) A_{s}^{j}, s \in\left[0, T_{2}\right]$, and $j=1,2, \ldots$, are positive i.i.d. semimartingales such that

$$
A_{0}^{j}=1 \quad \text { for all } j=1,2, \ldots
$$

Consider a European derivative written on the loss index with maturity $T_{2}$ and payoff $h\left(L_{T_{2}}\right)>0$ for a payoff function $h: \mathbb{R} \mapsto \mathbb{R}_{+}$. Since we have assumed that the interest rate $r$ is deterministic, without loss of generality, we can express the price process of the insurance derivative in discounted terms, i.e. we can set $r \equiv 0$. Then the price process of the option is given by

$$
\pi_{t}=\mathrm{E}\left[h\left(L_{T_{2}}\right) \mid \mathcal{F}_{t}\right], \quad t \in\left[0, T_{2}\right] .
$$

In the following we will calculate the expected payoff in (3.1) using Fourier transform techniques. To this end, we impose the following conditions.

(C1) The payoff function $h(\cdot)$ is continuous.

(C2) $h(\cdot)-k \in L^{2}(\mathbb{R})=\left\{g: \mathbb{R} \rightarrow \mathbb{C}\right.$ measurable $\left.\left.\left|\int_{-\infty}^{\infty}\right| g(x)\right|^{2} \mathrm{~d} x<\infty\right\}$ for some $k \in \mathbb{R}$.

Remark 3.1. In [1] we could have considered more general options that did not necessarily fulfill condition (C2) by considering dampened payoffs. However, the cost of this approach is that treating heavy-tailed distributions of claim sizes $Y$ becomes more complicated. The approach in this paper allows for general claim size modeling, including distributions with heavy tails. Furthermore, as we will see in Section 5, condition (C2) is, for example, satisfied by call and put spread catastrophe insurance options, the typical options traded in the market.

Now let

$$
\hat{h}(u)=\frac{1}{2 \pi} \int_{-\infty}^{\infty} \mathrm{e}^{-\mathrm{i} u z}(h(z)-k) \mathrm{d} z \quad \text { for all } u \in \mathbb{R}
$$

be the Fourier transform of $h(\cdot)-k$ and assume that

(C3) $\hat{h}(\cdot) \in L^{1}(\mathbb{R})$. 
Note that condition (C2) does not necessarily imply condition (C3). Since conditions (C2) and (C3) are in force, the following inversion formula holds (cf. [9, Section 8.2]):

$$
h(x)-k=\int_{-\infty}^{\infty} \mathrm{e}^{\mathrm{i} u x} \hat{h}(u) \mathrm{d} u .
$$

Remark 3.2. Note that the equality in (3.2) is everywhere, not only almost everywhere, because of condition (C1). If the probability distribution of $L_{T_{2}}$ had a Lebesgue density, an almost everywhere equality in (3.2) would have been sufficient for the following computations. However, since the loss index is driven by a compound Poisson process, the distribution of $L_{T_{2}}$ has atoms and we need an everywhere equality to guarantee (3.3), below.

By (3.2), and condition (C3), we obtain

$$
\begin{aligned}
\pi_{t} & =\mathrm{E}\left[h\left(L_{T_{2}}\right) \mid \mathcal{F}_{t}\right] \\
& =\mathrm{E}\left[h\left(L_{T_{2}}\right)-k \mid \mathcal{F}_{t}\right]+k \\
& =\mathrm{E}\left[\int_{-\infty}^{\infty} \exp \left\{\mathrm{i} u L_{T_{2}}\right\} \hat{h}(u) \mathrm{d} u \mid \mathcal{F}_{t}\right]+k \\
& =\int_{-\infty}^{\infty} \mathrm{E}\left[\exp \left\{\mathrm{i} u L_{T_{2}}\right\} \mid \mathcal{F}_{t}\right] \hat{h}(u) \mathrm{d} u+k,
\end{aligned}
$$

where in the last equation we could apply Fubini's theorem, because condition (C3) holds. Hence, in order to calculate the price process $\left(\pi_{t}\right)_{t \in\left[0, T_{2}\right]}$ in (3.4), the essential task is to compute the conditional characteristic function of $L_{T_{2}}$,

$$
c_{t}(u):=\mathrm{E}\left[\exp \left\{\mathrm{i} u L_{T_{2}}\right\} \mid \mathcal{F}_{t}\right]=\mathrm{E}\left[\exp \left\{\mathrm{i} u \sum_{j=1}^{N_{T_{1}}} Y_{j} A_{T_{2}-\tau_{j}}^{j}\right\} \mid \mathscr{F}_{t}\right], \quad u \in \mathbb{R}
$$

for $t \in\left[0, T_{2}\right]$. We define the conditional characteristic function of the reestimation martingale $A^{j}$ as

$$
\psi_{t}^{A^{j}}(s, u):=\mathrm{E}\left[\exp \left\{\mathrm{i} u A_{s}^{j}\right\} \mid \mathcal{F}_{t}^{A^{j}}\right], \quad 0 \leq t \leq s \leq T_{2},
$$

where $\mathcal{F}_{t}^{A^{j}}:=\sigma\left(A_{s}^{j}, 0 \leq s \leq t\right)$ is the filtration generated by the $j$ th reestimation factor. Then our main result is as follows.

Theorem 3.1. The conditional characteristic function, (3.5), of the loss index $L_{T_{2}}$ is given,

1. for $t<T_{1}$, by

$$
\begin{aligned}
c_{t}(u)= & \exp \left\{-\lambda\left(T_{1}-t\right)\left(1-\mathrm{E}\left[\psi_{0}^{A}\left(T_{2}-U, u Y\right)\right]\right)\right\} \\
& \times\left.\prod_{j=1}^{N_{t}} \psi_{t-s_{j}}^{A^{j}}\left(T_{2}-s_{j}, u y_{j}\right)\right|_{s_{j}=\tau_{j}, y_{j}=Y_{j}}, \quad u \in \mathbb{R} ;
\end{aligned}
$$

2. for $t \in\left[T_{1}, T_{2}\right]$, by

$$
c_{t}(u)=\left.\prod_{j=1}^{N_{T_{1}}} \psi_{t-s_{j}}^{A^{j}}\left(T_{2}-s_{j}, u y_{j}\right)\right|_{s_{j}=\tau_{j}, y_{j}=Y_{j}}, \quad u \in \mathbb{R} .
$$


Here $U$ is a uniformly distributed random variable on $\left[t, T_{1}\right]$ and $Y$ is a random variable with distribution function $F^{Y}$, independent of $U$.

Note that, in Theorem 3.1, the times of catastrophe occurrence, $\tau_{j}$, and the initial loss estimates, $Y_{j}$, up to time $t$ are known data.

\subsection{Proof of Theorem 3.1}

We distinguish the computations over the two periods.

1. For $t \in\left[0, T_{1}\right]$, we obtain, by hypothesis (H4) and by the independent increments of $N_{t}$,

$$
\begin{aligned}
c_{t}(u) & =\mathrm{E}\left[\exp \left\{\mathrm{i} u\left(\sum_{j=1}^{N_{t}} Y_{j} A_{T_{2}-\tau_{j}}^{j}+\sum_{j=N_{t}+1}^{N_{T_{1}}} Y_{j} A_{T_{2}-\tau_{j}}^{j}\right)\right\} \mid \mathcal{F}_{t}\right] \\
& =\underbrace{\mathrm{E}\left[\exp \left\{\mathrm{i} u \sum_{j=1}^{N_{t}} Y_{j} A_{T_{2}-\tau_{j}}^{j}\right\} \mid \mathcal{F}_{t}\right]}_{:=a_{t}(u)} \underbrace{\mathrm{E}\left[\exp \left\{\mathrm{i} u \sum_{j=N_{t}+1}^{N_{T_{1}}} Y_{j} A_{T_{2}-\tau_{j}}^{j}\right\} \mid \mathcal{F}_{t}\right]}_{:=b_{t}(u)} .
\end{aligned}
$$

We compute separately the terms $a_{t}$ and $b_{t}$ in (3.6). By hypothesis (H4) we have, for $a_{t}(u), u \in$ $\mathbb{R}$,

$$
\begin{aligned}
a_{t}(u) & =\mathrm{E}\left[\exp \left\{\mathrm{i} u \sum_{j=1}^{N_{t}} Y_{j} A_{T_{2}-\tau_{j}}^{j}\right\} \mid \mathcal{F}_{t}\right] \\
& =\mathrm{E}\left[\exp \left\{\mathrm{i} u \sum_{j=1}^{n} y_{j} A_{T_{2}-s_{j}}^{j}\right\} \mid \mathcal{F}_{t}\right]_{n=N_{t}, s_{j}=\tau_{j}, y_{j}=Y_{j}} \\
& =\prod_{j=1}^{N_{t}} \mathrm{E}\left[\exp \left\{\mathrm{i} u y_{j} A_{T_{2}-s_{j}}^{j}\right\} \mid \mathcal{F}_{t}\right]_{s_{j}=\tau_{j}, y_{j}=Y_{j}} \\
& =\prod_{j=1}^{N_{t}} \mathrm{E}\left[\exp \left\{\mathrm{i} u y_{j} A_{T_{2}-s_{j}}^{j}\right\} \mid \mathcal{F}_{t-s_{j}}^{A^{j}}\right]_{s_{j}=\tau_{j}, y_{j}=Y_{j}} \\
& =\left.\prod_{j=1}^{N_{t}} \psi_{t-s_{j}}^{A^{j}}\left(T_{2}-s_{j}, u y_{j}\right)\right|_{s_{j}=\tau_{j}, y_{j}=Y_{j}} .
\end{aligned}
$$

Note that, for $a_{t}(u)$, the $Y_{j} \mathrm{~s}$, the $\tau_{j} \mathrm{~s}$, and $N_{t}$ are known data, because the corresponding catastrophes happened before time $t$.

For the second term, $b_{t}(u), u \in \mathbb{R}$, we obtain, again by hypothesis (H4) and the independent increments of the Poisson process $N$,

$$
\begin{aligned}
& b_{t}(u) \\
& =\mathrm{E}\left[\exp \left\{\mathrm{i} u \sum_{j=N_{t}+1}^{N_{T_{1}}} Y_{j} A_{T_{2}-\tau_{j}}^{j}\right\} \mid \mathcal{F}_{t}\right] \\
& =\mathrm{E}\left[\exp \left\{\mathrm{i} u \sum_{j=N_{t}+1}^{N_{T_{1}}} Y_{j} A_{T_{2}-\tau_{j}}^{j}\right\}\right]
\end{aligned}
$$




$$
\begin{aligned}
& =\mathrm{E}\left[\mathrm{E}\left[\exp \left\{\mathrm{i} u \sum_{j=1}^{n} y_{j} A_{T_{2}-s_{j}}^{j}\right\} \mid N_{T_{1}}-N_{t}, Y_{1}, \tau_{1}, \ldots, \tau_{N_{T_{1}}-N_{t}}, \ldots, Y_{N_{T_{1}}-N_{t}}\right] \begin{array}{c}
n=N_{T_{1}}-N_{t} \\
y_{j}=Y_{j} \\
s_{j}=\tau_{j}
\end{array}\right] \\
& =\mathrm{E}\left[\mathrm{E}\left[\prod_{j=1}^{n} \psi_{0}^{A^{j}}\left(T_{2}-s_{j}, u y_{j}\right) \mid N_{T_{1}}-N_{t}, Y_{1}, \tau_{1}, \ldots, \tau_{N_{T_{1}}-N_{t}}, \ldots, Y_{N_{T_{1}}-N_{t}}\right] \begin{array}{c}
n=N_{T_{1}}-N_{t} \\
y_{j}=Y_{j} \\
s_{j}=\tau_{j}
\end{array}\right] \\
& =\mathrm{E}\left[\prod_{j=N_{t}+1}^{N_{T_{1}}} \psi_{0}^{A}\left(T_{2}-\tau_{j}, u Y_{j}\right)\right] .
\end{aligned}
$$

By Theorem 5.2.1 of [15] we obtain the following result.

Lemma 3.1. Let $N_{t}$ be a Poisson process with jump times $\tau_{j}, j=1,2, \ldots$ Then, for all $0 \leq t \leq T$,

$$
\left(\tau_{N_{t+1}}, \ldots, \tau_{N_{T}} \mid N_{T}-N_{t}=n\right)
$$

has the same distribution as the order statistics $\left(U_{(1)}, \ldots, U_{(n)}\right)$, where $U_{j}, j=1, \ldots, n$, are i.i.d. uniformly distributed on the interval $[t, T]$.

Using Lemma 3.1, and hypothesis (H4) again, we can replace the $\tau_{j} \mathrm{~s}$ in (3.7) with the order statistics $U_{(j)}$ of i.i.d. uniformly distributed random variables on the interval $\left[t, T_{1}\right]$ and obtain

$$
b_{t}(u)=\mathrm{E}\left[\prod_{j=N_{t}+1}^{N_{T_{1}}} \psi_{0}^{A}\left(T_{2}-U_{(j)}, u Y_{j}\right)\right], \quad u \in \mathbb{R} .
$$

Next, we note the following simple, helpful lemma.

Lemma 3.2. Consider the order statistics $U_{(1)}, \ldots, U_{(n)}$ of $n$ i.i.d. random variables $U_{1}, \ldots$, $U_{n}$ and a bounded measurable function $f\left(x_{1}, \ldots, x_{n}\right)$ symmetric in its arguments. Then

$$
\mathrm{E}\left[f\left(U_{(1)}, \ldots, U_{(n)}\right)\right]=\mathrm{E}\left[f\left(U_{1}, \ldots, U_{n}\right)\right] .
$$

Proof. We denote by $\Sigma_{n}$ the set of all permutations of $\{1, \ldots, n\}$. We have

$$
\begin{aligned}
\mathrm{E}\left[f\left(U_{(1)}, \ldots, U_{(n)}\right)\right] & =\mathrm{E}\left[\sum_{\sigma \in \Sigma_{n}} f\left(U_{\sigma(1)}, \ldots, U_{\sigma(n)}\right) \mathbf{1}_{\left\{U_{\sigma(1)}<\cdots<U_{\sigma(n)}\right\}}\right] \\
& =\mathrm{E}[f\left(U_{1}, \ldots, U_{n}\right) \underbrace{\left.\sum_{\sigma \in \Sigma_{n}} \mathbf{1}_{\left\{U_{\sigma(1)}<\cdots<U_{\sigma(n)}\right\}}\right]}_{1} \\
& =\mathrm{E}\left[f\left(U_{1}, \ldots, U_{n}\right)\right] .
\end{aligned}
$$

By the i.i.d. assumption of the $Y_{j}$ s and $A^{j}$ s, we see that the function

$$
f_{u}^{n}\left(s_{1}, \ldots, s_{n}\right):=\mathrm{E}\left[\prod_{j=1}^{n} \psi_{0}^{A}\left(T_{2}-s_{j}, u Y_{j}\right)\right], \quad u \in \mathbb{R}
$$


is symmetric in $s_{1}, \ldots, s_{n}$. It is then not difficult to see, using Lemma 3.2, that

$$
\begin{aligned}
b_{t}(u) & =\mathrm{E}\left[\mathrm{E}\left[\prod_{j=1}^{n} \psi_{0}^{A}\left(T_{2}-s_{j}, u Y_{j}\right) \mid N_{T_{1}}-N_{t}, U_{(1)}, \ldots, U_{\left(N_{T_{1}}-N_{t}\right)}\right]_{n=N_{T_{1}}-N_{t}, s_{j}=U_{(j)}}\right] \\
& =\mathrm{E}\left[\left.f_{u}^{n}\left(s_{1}, \ldots, s_{n}\right)\right|_{\left.n=N_{T_{1}}-N_{t}, s_{j}=U_{(j)}\right]}\right. \\
& =\mathrm{E}\left[\left.f_{u}^{n}\left(U_{(1)}, \ldots, U_{(n)}\right)\right|_{n=N_{T_{1}}-N_{t}}\right] \\
& =\mathrm{E}\left[\left.f_{u}^{n}\left(U_{1}, \ldots, U_{n}\right)\right|_{n=N_{T_{1}}-N_{t}}\right] \\
& =\mathrm{E}\left[\prod_{j=N_{t}+1}^{N_{T_{1}}} \psi_{0}^{A}\left(T_{2}-U_{j}, u Y_{j}\right)\right] \\
& =\mathrm{E}\left[\exp \left\{\mathrm{i} u \sum_{j=N_{t}+1}^{N_{T_{1}}} Y_{j} A_{T_{2}-U_{j}}^{j}\right\}\right],
\end{aligned}
$$

where we substituted the order statistics $U_{(j)}$ with the i.i.d. uniform variables $U_{j}$.

Note that (3.8) coincides with the characteristic function of a compound Poisson process of the form

$$
\sum_{j=N_{t}+1}^{N_{T_{1}}} Z^{j}
$$

where $Z^{j}=Y_{j} A_{T_{2}-U_{j}}^{j}, j=1,2, \ldots$, are i.i.d. The form of the characteristic function is in this case well known. Thus, we can rewrite (3.8) as

$$
\begin{aligned}
\mathrm{E}\left[\exp \left\{\mathrm{i} u \sum_{j=N_{t}+1}^{N_{T_{1}}} Y_{j} A_{T_{2}-U_{j}}^{j}\right\}\right] & =\exp \left\{-\lambda\left(T_{1}-t\right)\left(1-\mathrm{E}\left[\exp \left\{\mathrm{i} u Z^{1}\right\}\right]\right)\right\} \\
& =\exp \left\{-\lambda\left(T_{1}-t\right)\left(1-\mathrm{E}\left[\psi_{0}^{A}\left(T_{2}-U, u Y\right)\right]\right)\right\} .
\end{aligned}
$$

This completes the proof for the case in which $t \leq T_{1}$.

2. For the case in which $t>T_{1}$, we obtain

$$
c_{t}(u)=\left.\prod_{j=1}^{N_{T_{1}}} \psi_{t-s_{j}}^{A^{j}}\left(T_{2}-s_{j}, u y_{j}\right)\right|_{s_{j}=\tau_{j}, y_{j}=Y_{j}}, \quad u \in \mathbb{R},
$$

as for the term $a_{t}$ in the case in which $0 \leq t \leq T_{1}$.

This completes the proof of Theorem 3.1.

Remark 3.3. In [17] a special case of our model was presented, where the reestimation factor $A$ was geometric Brownian motion. In this case the conditional characteristic function of the reestimation factor can be computed by numeric integration by

$$
\begin{aligned}
\psi_{t}^{A}(s, u) & =\mathrm{E}\left[\exp \left\{\mathrm{i} u \exp \left\{B_{s}-\frac{1}{2} s\right\}\right\} \mid \mathcal{F}_{t}\right] \\
& =\mathrm{E}\left[\exp \left\{\mathrm{i} u \exp \left\{B_{t}-\frac{1}{2} t\right\}\right\} \exp \left\{B_{s}-B_{t}-\frac{1}{2}(s-t)\right\}\right] \\
& =\left.\mathrm{E}\left[\exp \left\{\mathrm{i} u w_{t} \exp \left\{B_{s-t}-\frac{1}{2}(s-t)\right\}\right\}\right]\right|_{w_{t}=\exp \left\{B_{t}-t / 2\right\}} \\
& =\left.\int \exp \left\{\mathrm{i} u w_{t} \mathrm{e}^{y}\right\} \exp \left\{-\frac{(y-(1 / 2)(s-t))^{2}}{2(s-t)}\right\} \mathrm{d} y\right|_{w_{t}=\exp \left\{B_{t}-t / 2\right\}}
\end{aligned}
$$


For further results about the characteristic function of lognormal random variables, we refer the reader to [10].

In the next section we turn our attention to a class of reestimation processes where the conditional characteristic function is numerically tractable and in some cases analytically obtainable: affine processes. For further information on affine processes and their applications to mathematical finance, we refer the reader to [5], [6], and [7].

\section{Reestimation with positive affine processes}

We suppose that the reestimation factors are given by positive affine processes. Affine processes constitute a reach class of processes suitable to model a wide range of phenomena. At the same time, the advantage is that the conditional characteristic function can be obtained explicitly up to the solution of two Riccati equations.

Definition 4.1. A Markov process $A=\left(A_{t}, \mathrm{P}_{x}\right)$ on $[0, \infty]$ is called an affine process if there exist $\mathbb{C}$-valued functions $\phi(t, u)$ and $\psi(t, u)$, defined on $\mathbb{R}_{+} \times \mathbb{R}$, such that

$$
\mathrm{E}\left[\exp \left\{\mathrm{i} u A_{T_{2}}\right\} \mid \mathcal{F}_{t}\right]=\exp \left\{\phi\left(T_{2}-t, u\right)+\psi\left(T_{2}-t, u\right) A_{t}\right\} \quad \text { for } t \geq 0 .
$$

We assume that

(A1) $A$ is conservative, i.e. for every $t>0$ and $x \geq 0$

$$
\mathrm{P}_{x}\left[A_{t}<\infty\right]=1
$$

(A2) $A$ is stochastically continuous for every $\mathrm{P}_{x}$.

By Proposition 1.1 of [8], assumption (A2) is equivalent to the assumption that $\phi(t, u)$ and $\psi(t, u)$ are continuous in $t$ for each $u$.

In the framework of our model, the computation of the conditional characteristic function reduces to the computation of $\phi$ and $\psi$. In some cases these are explicitly known, otherwise they can be obtained numerically. In the particular case when the reestimation factors remain positive affine martingales under the pricing measure, we are able to prove the following characterization, which provides a useful simplification of the conditional characteristic function.

Theorem 4.1. Let $A$ be an affine process satisfying assumptions (A1) and (A2). The affine process $A$ is a positive local martingale if and only if $A$ admits the following semimartingale characteristics $(B, C, v)$ :

$$
B_{t}=\beta \int_{0}^{t} A_{s} \mathrm{~d} s, \quad C_{t}=\alpha \int_{0}^{t} A_{s} \mathrm{~d} s, \quad \text { and } \quad v(\mathrm{~d} t, \mathrm{~d} y)=A_{t} \mu(\mathrm{d} y) \mathrm{d} t,
$$

where

$$
\beta=\mu[1, \infty)-\int_{1}^{\infty} y \mu(\mathrm{d} y)
$$

$\alpha \geq 0$, and $\mu$ is a Lévy measure on $(0, \infty)$.

Proof. Since $A_{t}$ satisfies assumptions (A1) and (A2), by [6, Theorem 2.12] and [8, Theorem 1.1], $A_{t}$ is a positive affine semimartingale if and only if $A_{t}$ admits the following characteristics $(B, C, v)$ :

$$
B_{t}=\int_{0}^{t}\left(\tilde{b}+\beta A_{s}\right) \mathrm{d} s, \quad C_{t}=\alpha \int_{0}^{t} A_{s} \mathrm{~d} s, \quad \text { and } \quad v(\mathrm{~d} t, \mathrm{~d} y)=\left(m(\mathrm{~d} y)+A_{t} \mu(\mathrm{d} y)\right) \mathrm{d} t,
$$


for every $\mathrm{P}_{x}$, where

$$
\tilde{b}=b+\int_{(0, \infty)}(1 \wedge y) m(\mathrm{~d} y),
$$

$\alpha, b \geq 0, \beta \in \mathbb{R}$, and $m$ and $\mu$ are Lévy measures on $(0, \infty)$, such that

$$
\int_{(0, \infty)}(y \wedge 1) m(\mathrm{~d} y)<\infty .
$$

See also [8]. By assumption (A2) and Theorem 7.16 of [3], the operator $L$,

$$
\begin{aligned}
L f(x)= & \frac{1}{2} \alpha x f^{\prime \prime}(x)+(b+\beta x) f^{\prime}(x)+\int_{(0, \infty)}(f(x+y)-f(x)) m(\mathrm{~d} y) \\
& +x \int_{(0, \infty)}\left(f(x+y)-f(x)-f^{\prime}(x)(1 \wedge y)\right) \mu(\mathrm{d} y)
\end{aligned}
$$

on $C^{2}\left(\mathbb{R}_{+}\right)$, is a version of the restriction of the extended infinitesimal generator of $A$ to $C^{2}\left(\mathbb{R}_{+}\right)$. (An operator $L$ with domain $\mathscr{D}_{L}$ is said to be an extended generator for $A$ if $\mathscr{D}_{L}$ consists of those Borel functions $f$ for which there exists a Borel function $L f$ such that the process

$$
L_{t}^{f}=f\left(A_{t}\right)-f\left(A_{0}\right)-\int_{0}^{t} L f\left(X_{s}\right) \mathrm{d} s
$$

is a local martingale.) Then $A$ is a local martingale if and only if

$$
L f(x) \equiv 0 \quad \text { for } f(x)=x .
$$

Substituting $f(x)=x$ into (4.2), we obtain

$$
\begin{aligned}
L x & =b+\beta x+\int_{(0, \infty)} y m(\mathrm{~d} y)+x \int_{(0, \infty)}(y-(1 \wedge y)) \mu(\mathrm{d} y) \\
& =\left(\beta+\int_{1}^{\infty}(y-1) \mu(\mathrm{d} y)\right) x+b+\int_{(0, \infty)} y m(\mathrm{~d} y) .
\end{aligned}
$$

Hence, $A$ is a local martingale if and only if

$$
\left(\beta+\int_{1}^{\infty}(y-1) \mu(\mathrm{d} y)\right) x+b+\int_{(0, \infty)} y m(\mathrm{~d} y)=0 \quad \text { for any } x \in \mathbb{R}_{+} .
$$

Since $b \geq 0$ and $m$ is a nonnegative measure, condition (4.3) means that

$$
b=0, \quad m \equiv 0, \quad \text { and } \quad \beta=\mu[1, \infty)-\int_{1}^{\infty} y \mu(\mathrm{d} y) .
$$

Let $A$ be an affine process satisfying assumptions (A1) and (A2). By Theorem 4.3 of [7], the conditional characteristic function of $A$ satisfies (4.1), where $\phi(t, u)$ and $\psi(t, u)$ solve the equations

$$
\begin{gathered}
\phi(t, u)=\int_{0}^{t} F(\psi(s, u)) \mathrm{d} s, \\
\partial_{t} \psi(t, u)=R(\psi(t, u)), \quad \psi(0, u)=\mathrm{i} u,
\end{gathered}
$$


where, for $z \in\{\mathbb{C} \mid \operatorname{Re} z \leq 0\}$,

$$
\begin{gathered}
R(z)=\frac{1}{2} \alpha z^{2}+\beta z+\int_{(0, \infty)}\left(\mathrm{e}^{z y}-1-z(y \wedge 1)\right) \mu(\mathrm{d} y), \\
F(z)=b z+\int_{(0, \infty)}\left(\mathrm{e}^{z y}-1\right) m(\mathrm{~d} y)
\end{gathered}
$$

and $\alpha, \beta, b, m$, and $\mu$ are parameters of infinitesimal generator (4.2) of $A$. If $A$ is a local martingale then by (4.4) we can simplify (4.7) and (4.8) as follows:

$$
\begin{gathered}
R(z)=\frac{1}{2} \alpha z^{2}+\int_{(0, \infty)}\left(\mathrm{e}^{z y}-z y-1\right) \mu(\mathrm{d} y), \\
F(z) \equiv 0 .
\end{gathered}
$$

From (4.5) and (4.10), we immediately obtain, for positive affine local martingales,

$$
\phi(t, u) \equiv 0 .
$$

In order to determine $\psi$, we have to, in general, solve (4.5) numerically. For some special cases, however, we can compute $\psi$ analytically. We give two examples.

Example 4.1. If $A$ has no jump part then $A$ is called a Feller diffusion (see, e.g. [6]). In this case the positive affine martingale dynamics are given by

$$
\mathrm{d} A_{t}=\sqrt{\alpha A_{t}} \mathrm{~d} W_{t},
$$

where $W_{t}$ is a standard Brownian motion. Consequently, we have $\mu=0$ in (4.9), and we can rewrite (4.6) as

$$
\psi_{t}^{\prime}=\frac{1}{2} \alpha \psi_{t}^{2} .
$$

Solving the differential equation (4.11), we obtain

$$
\psi(t, u) \equiv 0 \quad \text { or } \quad \psi(t, u)=-\frac{1}{\alpha t / 2+C(u)}, \quad u \in \mathbb{R},
$$

where $C(u)$ can be found from the boundary condition $\psi(0, u)=\mathrm{i} u$. Substituting $C(u)$ into the expression for $\psi$, we obtain

$$
\psi(t, u) \equiv 0 \quad \text { or } \quad \psi(t, u)=-\frac{1}{\alpha t / 2+\mathrm{i} / u}, \quad u \in \mathbb{R} .
$$

Note that if we have no jump part then $A$ has positive probability of being absorbed at 0 . However, it may still be of interest to also consider the case of positive probability of absorption at 0 , if we wish to include the possibility of fraud or falsified reporting of claims into the model. In this case, reestimation might discover the fraud and the previous fake evaluation will be set to 0 .

Example 4.2. In order to give an example of a positive affine martingale including jumps where we can solve for $\psi$ explicitly, we specify the jump density $\mu(\mathrm{d} y)$ in the semimartingale characteristics in Theorem 4.1 as

$$
\mu(\mathrm{d} y)=\frac{3}{4 \sqrt{\pi}} \frac{\mathrm{d} y}{y^{5 / 2}} .
$$


Then some calculations give $R(z)$ in (4.7):

$$
\begin{aligned}
R(z) & =\frac{1}{2} \alpha z^{2}+\frac{3}{4 \sqrt{\pi}} \int_{(0, \infty)}\left(\mathrm{e}^{z y}-z y-1\right) \frac{\mathrm{d} y}{y^{5 / 2}} \\
& =\frac{1}{2} \alpha z^{2}+(-z)^{3 / 2} \quad \text { for } z \in\{\mathbb{C} \mid \operatorname{Re} z \leq 0\} .
\end{aligned}
$$

Consider $\eta(t, u):=-\psi(t, u)$. By (4.6) we then have

$$
-\eta_{t}^{\prime}=\frac{1}{2} \alpha \eta^{2}+\eta^{3 / 2}
$$

The solutions to (4.12) are $\eta(t, u) \equiv 0$ and

$$
\eta(t, u)=\frac{4}{\alpha^{2}}\left(1+W\left(-C(u) \mathrm{e}^{-t / \alpha}\right)\right)^{-2},
$$

where $W(\cdot)$ is the Lambert $W$ function. (The Lambert $W$ function, $W(z)$, is defined to be the function satisfying $W(z) \mathrm{e}^{W(z)}=z, z \in \mathbb{C}$. See also [4] for more details on the Lambert function.) The boundary condition $\eta(0, u)=-\psi(0, u)=\mathrm{i} u$ yields

$$
C(u)=-\left(-1+\frac{2}{\alpha} \sqrt{\frac{\mathrm{i}}{u}}\right) \exp \left\{-1+\frac{2}{\alpha} \sqrt{\frac{\mathrm{i}}{u}}\right\} .
$$

Substituting $C(u)$ into (4.13), we obtain, for $\psi(t, u)=-\eta(t, u)$,

$$
\psi(t, u) \equiv 0 \quad \text { or } \quad \psi(t, u)=-\frac{4}{\alpha^{2}}\left(1+W\left(\left(-1+\frac{2}{\alpha} \sqrt{\frac{\mathrm{i}}{u}}\right) \exp \left\{-\frac{t}{\alpha}-1+\frac{2}{\alpha} \sqrt{\frac{\mathrm{i}}{u}}\right\}\right)\right)^{-2} .
$$

\section{Pricing of call and put spreads}

We conclude this paper by showing that we can apply the developed pricing method to spread options, which are the typical catastrophe options traded in the market. A call spread option with strike prices $0<K_{1}<K_{2}$ is a European derivative with the payoff function at maturity given by

$$
h(x)= \begin{cases}0 & \text { if } 0 \leq x \leq K_{1}, \\ x-K_{1} & \text { if } K_{1}<x \leq K_{2}, \\ K_{2}-K_{1} & \text { if } x>K_{2}\end{cases}
$$

The integrability condition $h(\cdot)-k \in L^{2}\left(\mathbb{R}_{+}\right)$is satisfied for $k:=K_{2}-K_{1}$. In particular, $h(\cdot)-k \in L^{1}\left(\mathbb{R}_{+}\right)$.

To satisfy conditions (C1) and (C3), we continuously extend $h$ from $\mathbb{R}_{+}$to $\mathbb{R}$ by

$$
\bar{h}(x):= \begin{cases}h(-x) & \text { if } x<0 \\ h(x) & \text { if } x \geq 0\end{cases}
$$

Note that the price processes of the two corresponding options with payoffs $h\left(L_{T_{2}}\right)$ and $\bar{h}\left(L_{T_{2}}\right)$ remain the same, because $L_{T_{2}} \geq 0$.

Let

$$
\hat{\bar{h}}(u):=\frac{1}{2 \pi} \int_{-\infty}^{\infty} \mathrm{e}^{-\mathrm{i} u z}(\bar{h}(z)-k) \mathrm{d} z \quad \text { for all } u \in \mathbb{R}
$$


be the Fourier transform of $\bar{h}-k$. Then

$$
\begin{aligned}
\hat{\bar{h}}(u)= & \frac{1}{2 \pi}\left(\int_{-K_{2}}^{-K_{1}} \mathrm{e}^{-\mathrm{i} u x}\left(-x-K_{2}\right) \mathrm{d} x+\int_{-K_{1}}^{K_{1}} \mathrm{e}^{-\mathrm{i} u x}\left(K_{1}-K_{2}\right) \mathrm{d} x\right. \\
& \left.\quad+\int_{K_{1}}^{K_{2}} \mathrm{e}^{-\mathrm{i} u x}\left(x-K_{2}\right) \mathrm{d} x\right) \\
= & \frac{1}{2 \pi} \frac{1}{u^{2}}\left(\exp \left\{-\mathrm{i} u K_{2}\right\}+\exp \left\{\mathrm{i} u K_{2}\right\}-\exp \left\{-\mathrm{i} u K_{1}\right\}-\exp \left\{\mathrm{i} u K_{1}\right\}\right) \\
= & \frac{1}{\pi} \frac{1}{u^{2}}\left(\operatorname{Re}\left(\exp \left\{\mathrm{i} u K_{2}\right\}\right)-\operatorname{Re}\left(\exp \left\{\mathrm{i} u K_{1}\right\}\right)\right) \\
= & \frac{1}{\pi} \frac{1}{u^{2}}\left(\cos u K_{2}-\cos u K_{1}\right) \in L^{1}(\mathbb{R}),
\end{aligned}
$$

and, by applying the inversion formula (3.2) to $\bar{h}(x)$ for $x \geq 0$, we find that (3.2) also holds for $h$, since $h(x)=\bar{h}(x)$ for $x \geq 0$.

In particular, since $L_{T_{2}} \geq 0$ almost surely for the price of the call spread, we can write

$$
\begin{aligned}
\pi_{t}^{\mathrm{CS}} & =\mathrm{E}\left[h\left(L_{T_{2}}\right)-k \mid \mathcal{F}_{t}\right]+k \\
& =\mathrm{E}\left[\bar{h}\left(L_{T_{2}}\right)-k \mid \mathcal{F}_{t}\right]+k \\
& =\mathrm{E}\left[\int_{-\infty}^{\infty} \exp \left\{\mathrm{i} u L_{T_{2}}\right\} \hat{\bar{h}}(u) \mathrm{d} u \mid \mathcal{F}_{t}\right]+k \\
& =\int_{-\infty}^{\infty} \mathrm{E}\left[\exp \left\{\mathrm{i} u L_{T_{2}}\right\} \mid \mathcal{F}_{t}\right] \hat{\bar{h}}(u) \mathrm{d} u+k \\
& =\int_{-\infty}^{\infty} c_{t}(u) \hat{\bar{h}}(u) \mathrm{d} u+k \\
& =\frac{1}{\pi} \int_{-\infty}^{\infty} \frac{c_{t}(u)}{u^{2}}\left(\cos u K_{2}-\cos u K_{1}\right) \mathrm{d} u+K_{2}-K_{1},
\end{aligned}
$$

where $c_{t}(u)$ is defined in (3.4). Note that the integral in (5.1) is real, since $\operatorname{Im}\left(c_{t}(-u)\right)=$ $-\operatorname{Im}\left(c_{t}(u)\right)$ by definition of $c_{t}$.

Analogously, for the put spread catastrophe option with payoff at the maturity given by

$$
g(x)= \begin{cases}K_{2}-K_{1} & \text { if } 0 \leq x \leq K_{1} \\ K_{2}-x & \text { if } K_{1}<x \leq K_{2} \\ 0 & \text { if } x>K_{2}\end{cases}
$$

we obtain

$$
\pi_{t}^{\mathrm{PS}}=\frac{1}{\pi} \int_{-\infty}^{\infty} \frac{c_{t}(u)}{u^{2}}\left(\cos u K_{1}-\cos u K_{2}\right) \mathrm{d} u .
$$

Note that the call-put parity is satisfied:

$$
\pi_{t}^{\mathrm{PS}}=K_{2}-K_{1}-\pi_{t}^{\mathrm{CS}}
$$

\section{Acknowledgements}

We wish to thank the New York Mercantile Exchange for the information provided concerning catastrophe insurance options and Damir Filipović for interesting comments and remarks. 


\section{References}

[1] Biagini, F., Bregman, Y. and Meyer-Brandis, T. (2008). Pricing of catastrophe insurance options written on a loss index. To appear in Insurance Math. Econom.

[2] Christensen, C. V. (1999). A new model for pricing catastrophe insurance derivatives. CAF Working Paper Series No. 28.

[3] Çinlar, E., Jacod, J., Protter, P. and Sharpe, M. J. (1980). Semimartingales and Markov processes. Prob. Theory Relat. Fields 54, 161-219.

[4] Corless, R. M. et al. (1996). On the Lambert $W$ function. Adv. Comput. Math. 5, 329-359.

[5] Duffie, D., PAN, J. And Singleton, K. (2000). Transform analysis and asset pricing for affine jump diffusions. Econometrica 68, 1343-1376.

[6] Duffie, D., Filipovic, D. And Schachermayer, W. (2003). Affine processes and applications in finance. Ann. Appl. Prob. 13, 984-1053.

[7] Filipović, D. (2001). A general characterization of one factor affine term structure models. Finance Stoch. 5, 389-412.

[8] KaVAZU, K. and Watanabe, S. (1971). Branching processes with immigration and related limit theorems. Theory Prob. Appl. 16, 36-54.

[9] Königsberger, K. (1993). Analysis 2. Springer, Berlin.

[10] LeIPNIK, R. B. (1991). On lognormal random variables. I. The characteristic function. J. Austral. Math. Soc. Ser. B 32, 327-347.

[11] MöLler, M. (1996). Pricing PCS-options with the use of Esscher-transforms. AFIR Colloquium Nürnberg, October 1-3 1996. Available at http://www.actuaries.org/AFIR/colloquia/Nuernberg/papers.cfm.

[12] Mürmann, A. (2001). Pricing catastrophe insurance derivatives. Financial Markets Group Discussion Paper 400. Available at http://ssrn.com/abstract $=31052$.

[13] Mürmann, A. (2003). Actuarially consistent valuation of catastrophe derivatives. Working paper. Available at http://knowledge.wharton.upenn.edu/muermann/.

[14] Mürmann, A. (2006). Market price of insurance risk implied by catastrophe derivatives. Working paper. Available at http://irm.wharton.upenn.edu/muermann/.

[15] Rolski, T., Schmidli, H., Schmidt, V. And Teugles, J. L. (1999). Stochastic Processes for Insurance and Finance. John Wiley, Chichester.

[16] SchmidLI, H. (2001). Modeling PCS options via individual indices. Working paper 187, Laboratory of Actuarial Mathematics, University of Copenhagen.

[17] Schradin, H. R. (1996). PCS Catastrophe insurance options-a new instrument for managing catastrophe risk. Contribution to the 6th AFIR International Colloquium Nürnberg, October 1-3 1996. Available at http://www.actuaries.org/AFIR/colloquia/Nuernberg/papers.cfm.

[18] Sigma NR. 3 (2001). Capital Market Innovation in the Insurance Industry. Swiss Re, Zürich.

[19] Sigma Nr. 7 (2006). Securitization-New Opportunities for Insurers and Investors. Swiss Re, Zürich.

[20] Sigma Nr. 2 (2007). Natural Catastrophes and Man-Made Disasters in 2006. Swiss Re, Zürich. 